\title{
Effect of media distraction (audio-visual and music) for pain and anxiety control in patients undergoing shock-wave lithotripsy: A systematic review and meta-analysis
}

\author{
WEIHUA HU ${ }^{1}, \mathrm{KE} \mathrm{YANG}^{1}, \mathrm{LI}_{\mathrm{ZHANG}}{ }^{1}$ and $\mathrm{XU} \mathrm{LU}^{2}$ \\ Departments of ${ }^{1}$ Urology and ${ }^{2}$ Laboratory Hunan Provincial People's Hospital, \\ The First Affiliated Hospital of Hunan Normal University, Changsha, Hunan 410000, P.R. China
}

Received April 27, 2020; Accepted January 20, 2021

DOI: $10.3892 /$ etm.2021.10055

\begin{abstract}
Audio-visual (AV) or music distraction may be used to reduce pain during several healthcare procedures. The present manuscript is a systematic review and meta-analysis to assess the effectiveness of media distraction in reducing pain and anxiety in extracorporeal shock wave lithotripsy (ESWL) patients. The PubMed, Embase, Scopus, BioMed Central, Ovoid and CENTRAL (Cochrane Central Register of Controlled Trials) databases were screened for studies assessing the role of media distraction (music/AV media) in reducing pain and anxiety of ESWL patients. Data were summarized using the mean difference (MD) with $95 \%$ confidence intervals (CI). A total of 11 randomized controlled trials were included. Pooled analysis indicated a statistically significant difference in pain outcomes with media distraction [mean difference (MD): -1.18; 95\% CI: $-2.35,-0.01 ; \mathrm{I}^{2}=96.8 \%$ )]. Subgroup analysis indicated that both AV media (MD: -2.94; 95\% CI: $-4.70,-1.17 ; \mathrm{I}^{2}=79.2 \%$ ) and music (MD: $-0.86 ; 95 \% \mathrm{CI}$ : $-1.37,-0.35 ; \mathrm{I}^{2}=62.5 \%$ ), led to significant reduction in pain outcomes. Pooled analysis indicated a statistically significant reduction of anxiety scores with the use of media distraction (MD: -3.91 ; 95\% CI: $-6.44,-1.38 ; \mathrm{I}^{2}=77.7 \%$ ). To conclude, the present review suggests that media distraction in the form of AV media or music may be beneficial in reducing the pain and anxiety of patients undergoing ESWL. Evidence is, however, weak considering the small effect size, confidence intervals being close to zero, and instability of the results on sensitivity analysis. In clinical practice, media distraction may be used during ESWL as a nursing intervention, but a clinically important reduction of pain and anxiety may not be expected.
\end{abstract}

Correspondence to: $\mathrm{Mr}$. Xu Lu, Department of Laboratory, Hunan Provincial People's Hospital, The First Affiliated Hospital of Hunan Normal University, Room 202, East Building 15-Luqiao Group, Shaoshan South Road, Yuhua, Changsha, Hunan 410000, P.R. China E-mail: luxusky@126.com

Key words: music, audiovisual, distraction, complementary therapy, analgesia, urology, renal stones

\section{Introduction}

Urinary stones are a significant health problem affecting $\sim 12 \%$ of the population in North America, 5-9\% in Europe and $1-5 \%$ in Asia (1). Analysis of global trends indicates that due to climate change, obesity and dietary modifications, the worldwide prevalence of urinary stones is gradually increasing (2). Since the 1980s, extracorporeal shock wave lithotripsy (ESWL) has been widely used in the management of urinary tract calculi, due to its high efficacy and low morbidity (3). After the manufacture of the first ESWL device in 1984, several modifications have made subsequent devices smaller and less powerful, allowing the management of urinary stones without the use of anesthetic (4,5). Despite technological development, $30 \%$ of patients still complain of severe pain (6). Pain during ESWL can affect the patients' tolerance of the procedure as well as the effectiveness of treatment (7). Clinicians have reportedly used a number of pain control agents, including opioids, non-steroidal anti-inflammatory drugs (NSAIDs), nitrous oxide, local anesthetic infiltration and dermal anesthetics such as Emla, to manage the analgesic requirements of ESWL (6).

Nursing personnel play an important role in patient preparation and management of the patients' pain and anxiety (8). They are not only responsible for prescription drug administration but can also support pain management by a variety of complementary therapies $(9,10)$. In the world of modern media, audio-visual (AV) or music distraction has been successfully used to reduce procedural pain for many non-urological procedures (11-14). Considering the widespread use of the ESWL procedure, a number of researchers have also studied the efficacy of such media distraction techniques for pain management in urinary stone patients $(15,16)$. A recent meta-analysis from Kyriakides et al (15) evaluated the role of music in reducing pain in patients undergoing urological procedures. Their study, updated to 2017, included only six trials on patients undergoing ESWL. Their review was focused on only music therapy and did not present results of studies only on ESWL. Considering this lacuna in the literature, the purpose of the present study was to conduct a systematic review and meta-analysis to assess the effectiveness of any media distraction in reducing pain and anxiety of ESWL patients. 


\section{Materials and methods}

Inclusion criteria. The Preferred Reporting Items for Systematic Reviews and Meta-analyses (PRISMA) guidelines (17) and guidelines from the Cochrane collaboration (18) were followed during the conduct of this systematic review. The Population, Intervention, Comparison, Outcome and Study design (PICOS) model was used to select studies for inclusion in the review (17). Studies conducted on patients undergoing ESWL (population) were considered for inclusion. All randomized controlled trials (RCTs) comparing any type of media distraction (music/AV media) used during the ESWL procedure (intervention), with control (comparison) and measuring pain and/or anxiety (outcomes) were included. Studies were included irrespective of sample size and language of publication. The following studies were excluded: i) Studies utilizing a combination of music/AV media with any other complementary therapy, including relaxation therapy, acupuncture and guided imagery, in the study group; ii) studies utilizing music/AV media for any procedures other than ESWL; iii) studies not reporting pain and/or anxiety as an outcome measure; iv) non-RCTs; v) studies not comparing music/AV media with a control and vi) review articles, cases series and case reports. In the case of duplication of data, the most updated version of the study was to be included.

Search strategy. An electronic search was performed independently by two reviewers. The PubMed, Embase, Scopus, BioMed Central, Ovoid, and Cochrane Central Register of Controlled Trials (CENTRAL) databases were screened up to 15th February 2020. Both MeSH terms and free-text keywords were utilized for searching relevant articles. Search terms and search strategy along with the results of the PubMed database are presented in Table SI. The reviewers screened the search results initially by their titles and abstracts for every database. After identifying potentially pertinent articles, full texts of the articles were sourced. Both the reviewers assessed individual articles based on the inclusion and exclusion criteria. Any disagreements were resolved by discussion. Post-screening, the bibliography of included studies, as well as review articles on the subject, were hand searched for any additional references.

Data extraction and risk of bias assessment. The two reviewers independently extracted data from the included studies. Data regarding authors, publication year, study type, inclusion/exclusion criteria, sample size, demographic details, types of AV media, number of shock waves, ESWL power settings, use of analgesics and study outcomes were extracted. The authors were contacted by e-mail for any missing data. The primary outcome of the analysis was pain scores recorded after the ESWL procedure using the visual analog scale (VAS). The secondary outcome of interest was anxiety measured by the State and Trait Anxiety Inventory-State Anxiety (STAI-SA) test (19).

The Cochrane Collaboration risk assessment tool was used to assess the quality of included studies (18). Studies were assessed by two reviewers independently for: Random sequence generation, allocation concealment, blinding of participants and personnel, blinding of outcome assessment, incomplete outcome data, selective reporting and other bias.
Statistical analysis. The software 'Open MetaAnalyst (Version 10.10)' was used for the meta-analysis (20). A meta-analysis was conducted only if at least three studies reported data on the same outcome variable. Predicting heterogeneity in the included studies, a random-effects model was used to calculate the pooled effect size for all analyses. Heterogeneity was assessed using the $\mathrm{I}^{2}$ statistic. $\mathrm{I}^{2}$ values of $25-50 \%$ represented low, values of $50-75 \%$ medium and $>75 \%$ represented substantial heterogeneity. Continuous data were summarized using the mean difference (MD) with 95\% confidence intervals (CI). Sub-group analysis was performed for the use of music or $\mathrm{AV}$ media and the use of noise-canceling headphones for the primary outcome. For studies not reporting mean and standard deviation scores of outcome variables, the same was calculated from median and interquartile range using methods reported by Wan et al (21). A sensitivity analysis was carried out to assess the influence of each study on the pooled effect size. In this analysis, data from the various included studies were excluded one at a time and the effect size was recalculated. Due to the inclusion of fewer than 10 studies in the review, funnel plots were not used to assess publication bias (18).

\section{Results}

Study characteristics. A total of 438 unique records were screened and 13 articles were selected for full-text review (Fig. 1). Finally, 11 articles were included in this systematic review and meta-analysis (22-32). Details of the included studies are presented in Table I. Amongst all RCTs, only one was a cross-over trial (27). The sample size per arm in the included studies varied from 21-200 patients. In the study of Karalar et al (28), the study sample was further subdivided into two groups, based on the use of noise cancellation headphones. Details of these sub-groups were pooled separately. El-Khoury et al (32) conducted a three-arm trial comparing AV media and music with control. The data of these study groups were pooled separately for the meta-analysis. Çift and Benlioglu (24) in a five-arm study, compared three different types of music with two control groups of which one was provided with headphones while the other was not. The results of the study and control groups were pooled using a random-effects meta-analysis model into a single group (18). While different types of music were used in the included studies, two studies used AV media during ESWL. In the study of Marsdin et al (22), patients were provided with DVDs and television sets, while in the trial of El-Khoury et al (32) an iPad with music videos was used. Media distraction was delivered via headphones in all trials. A total of three studies reported the use of noise-cancellation headphones $(28,30,32)$. In studies reporting adequate data, there was no statistically significant difference in the number of shock waves and the power setting of ESWL between the study and control groups. Seven studies reported the use of analgesic/anxiolytics during the trial $(22,23,25,26,28,30,32)$. In the trial of Yilmaz et al (26), midazolam was used only in the control group whereas in all remaining studies drugs were not restricted to any specific group.

The authors' assessment of the quality of included studies is presented in Table II. Appropriate methods of randomization were not used in majority studies. Due to the nature of 


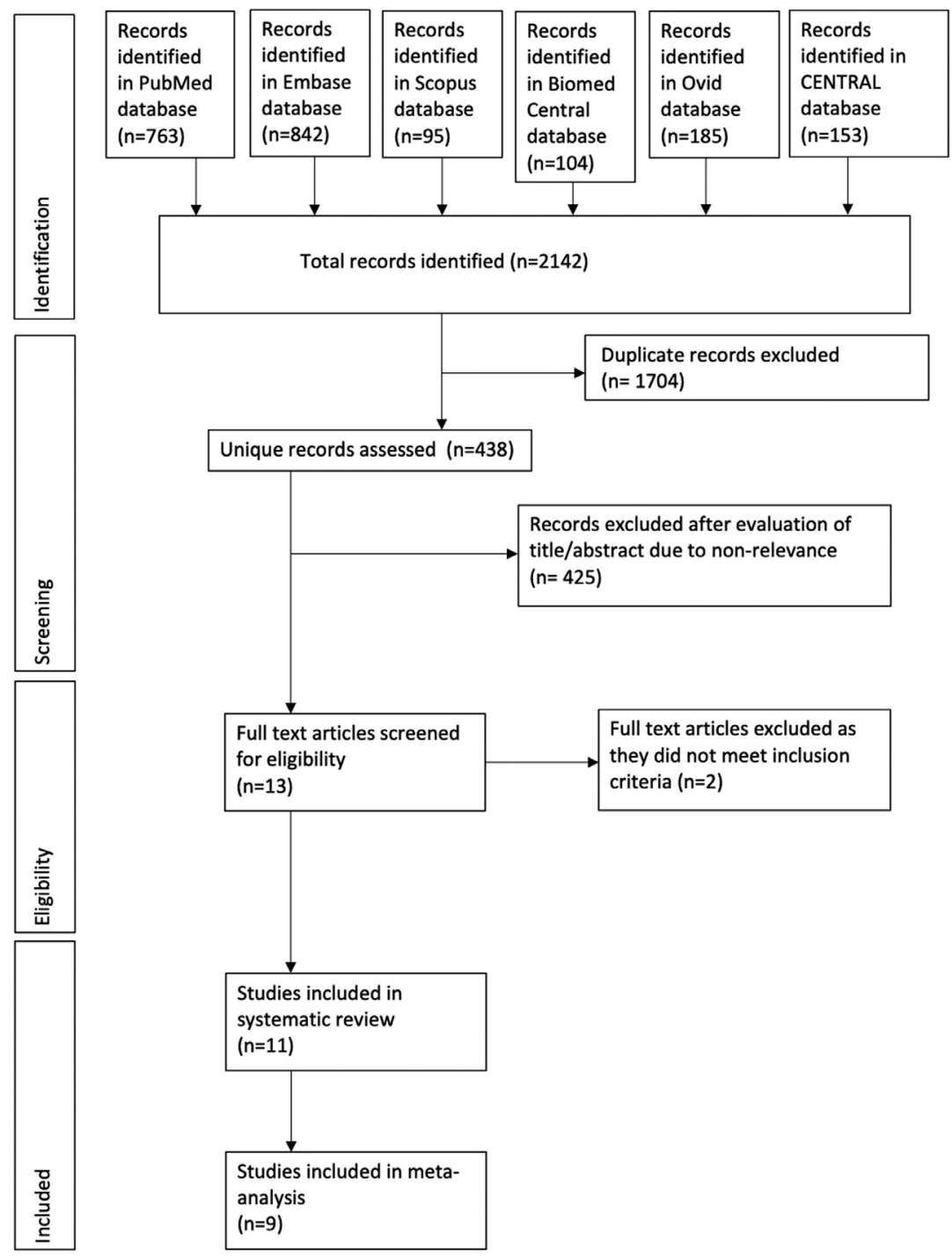

Figure 1. Study flow-chart.

the intervention, blinding of patients was not possible. The use of analgesics, which could have introduced bias in pain outcomes, was taken into consideration for 'other bias'.

Outcomes. A total of nine studies reported pain outcomes on the VAS scale $(22-24,26,28-32)$. Pooled analysis indicated a statistically significant difference in pain outcomes in favor of media distraction (MD: -1.18 ; 95\% CI: $-2.35,-0.01 ; \mathrm{I}^{2}=96.8 \%$; Fig. 2). On subgroup analysis based on the type of media distraction, the use of both, AV media (MD: $-2.94 ; 95 \% \mathrm{CI}$ : $-4.70,-1.17 ; \mathrm{I}^{2}=79.2 \%$ ) and music (MD: $-0.86 ; 95 \% \mathrm{CI}:-1.37$, $-0.35 ; \mathrm{I}^{2}=62.5 \%$ ), was indicated to lead to a significant reduction in pain outcomes (Fig. 2). Results were, however, not stable on sensitivity analysis. On the exclusion of the studies of Karalar et al (28) and El-Khoury et al (32), no significant effect of media distraction on pain outcomes was identified (Fig. 3). Data from study groups using noise-cancellation headphones were pooled separately in a sub-group analysis. The results indicated that there was a statistically significant reduction in pain scores with the use of noise-cancellation headphones (MD: -1.20 ; 95\% CI: $-2.17,-0.23 ; \mathrm{I}^{2}=69.8$ ) but no such effect was seen with regular headphones (MD: -1.13 ; 95\% CI: -2.66, 0.40; $\mathrm{I}^{2}=97.2 \%$ ) (Fig. 4).

There were two studies that did not report pain outcomes as mean \pm standard deviation. E-mail to the corresponding author for these missing data did not elicit a response. In the trial of Cepeda et al (25), alfentanil consumption was compared in the study and control groups. The authors reported no statistically significant difference in analgesic use and pain intensity during ESWL in the two groups. Akbas et al (27), on the other hand, in a cross-over RCT, reported a statistically significant reduction of pain with the use of music after both treatment sessions.

Anxiety outcomes using STAI-SA were reported by five studies $(24,26,28,29,31)$. All studies had used only music in the study groups. Pooled analysis indicated a statistically significant reduction of anxiety scores with the use of media distraction (MD: -3.91 ; 95\% CI: $-6.44,-1.38$; $\mathrm{I}^{2}=77.7 \%$; Fig. 5). The results were stable on sensitivity analysis as there was no change in significance of the results on exclusion of one study at a time (Fig. 6). Exclusion of the study of Yilmaz et al (26) used midazolam in the control group and the "noise cancellation headphone' group of Karalar et al (28) did not change the significance of results. Ordaz Jurado et al (30) compared anxiety 


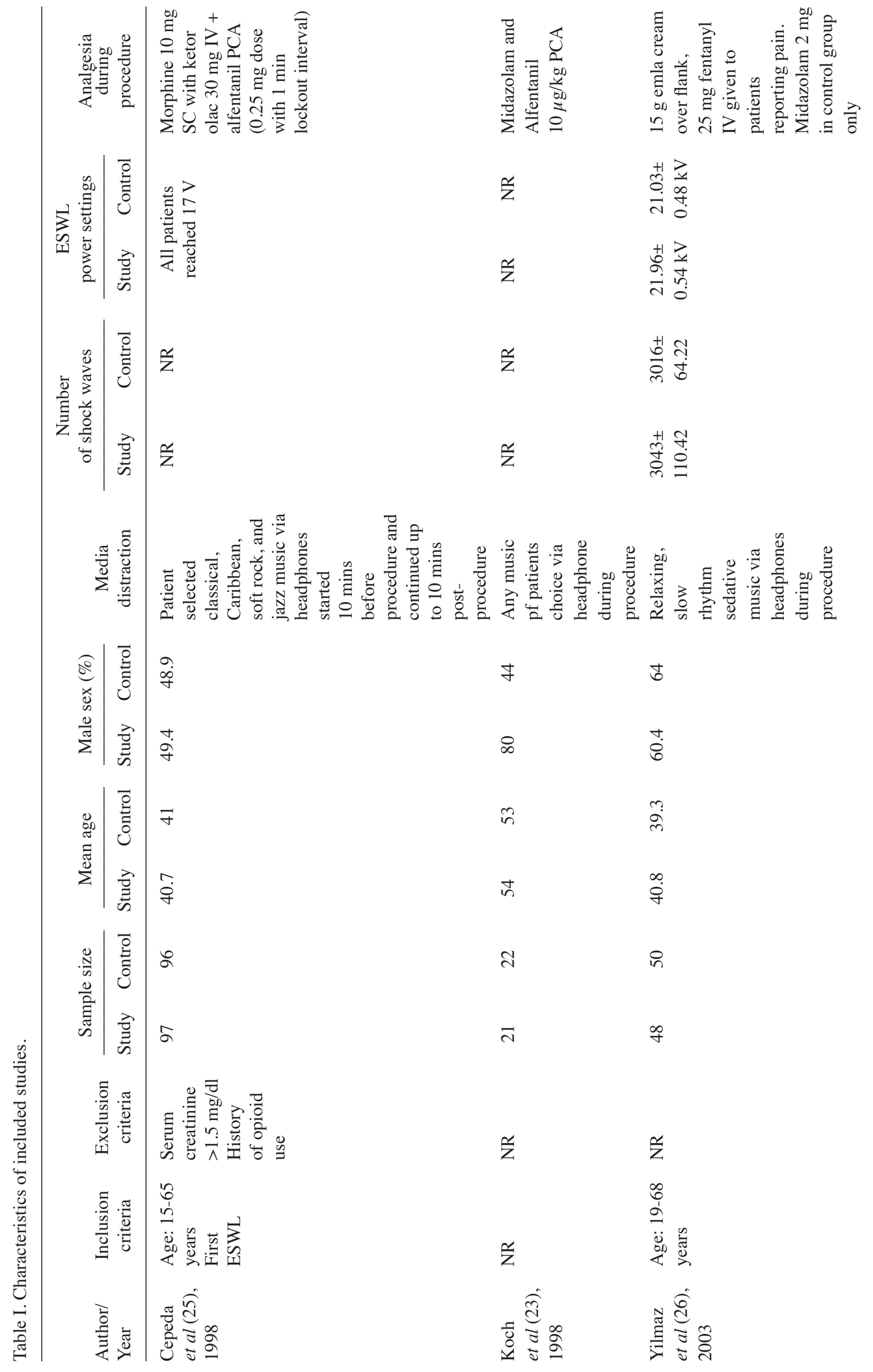




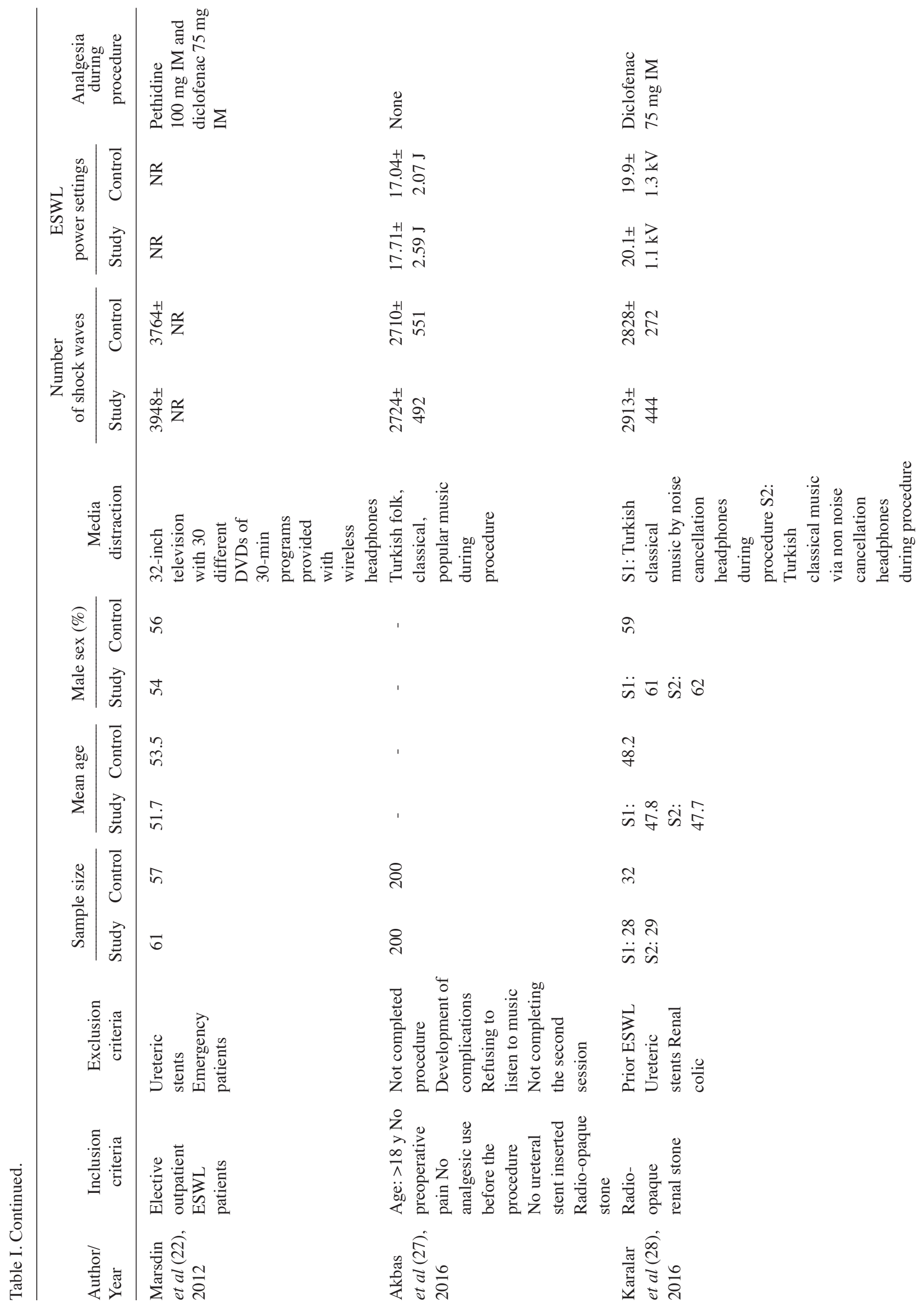




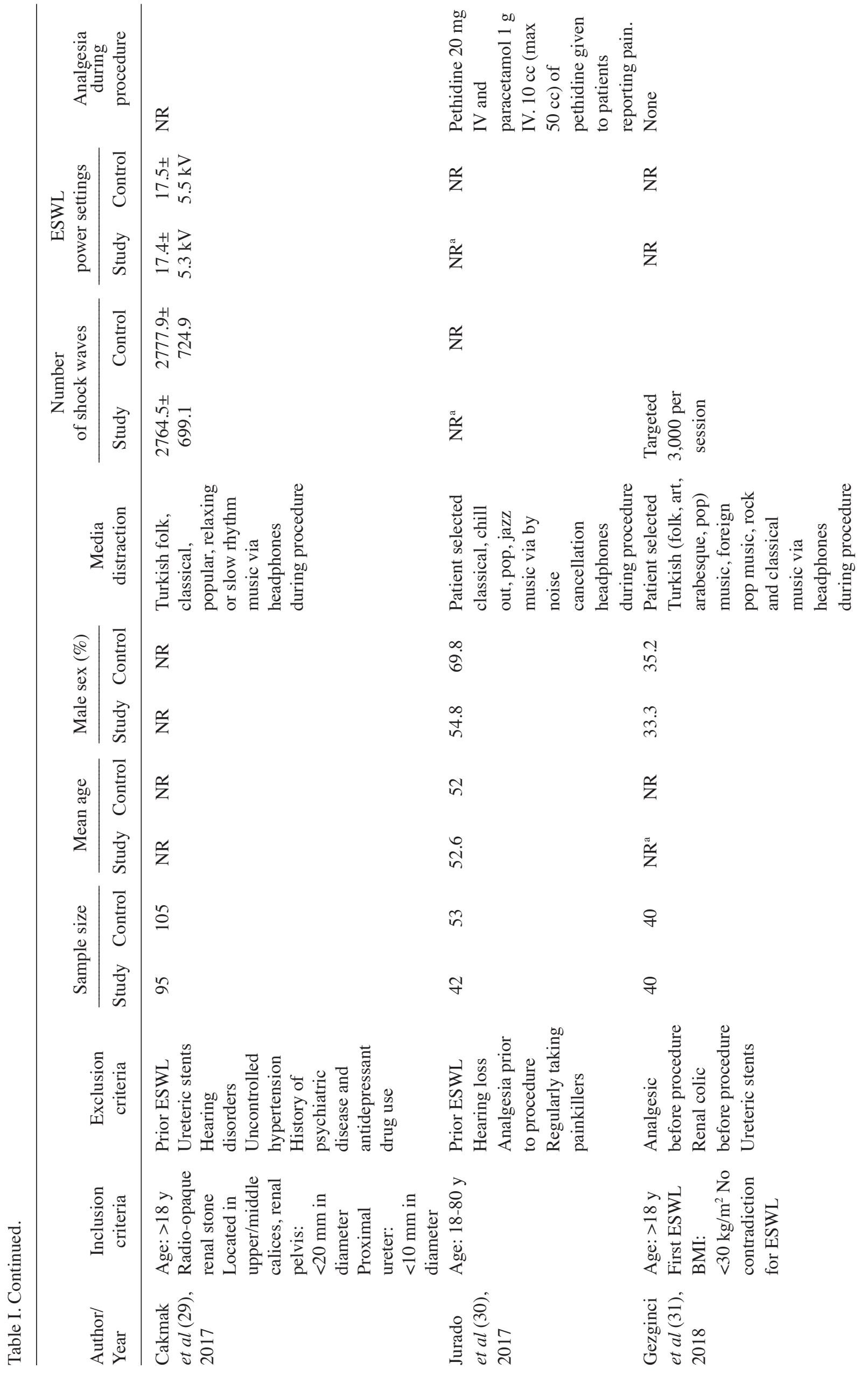




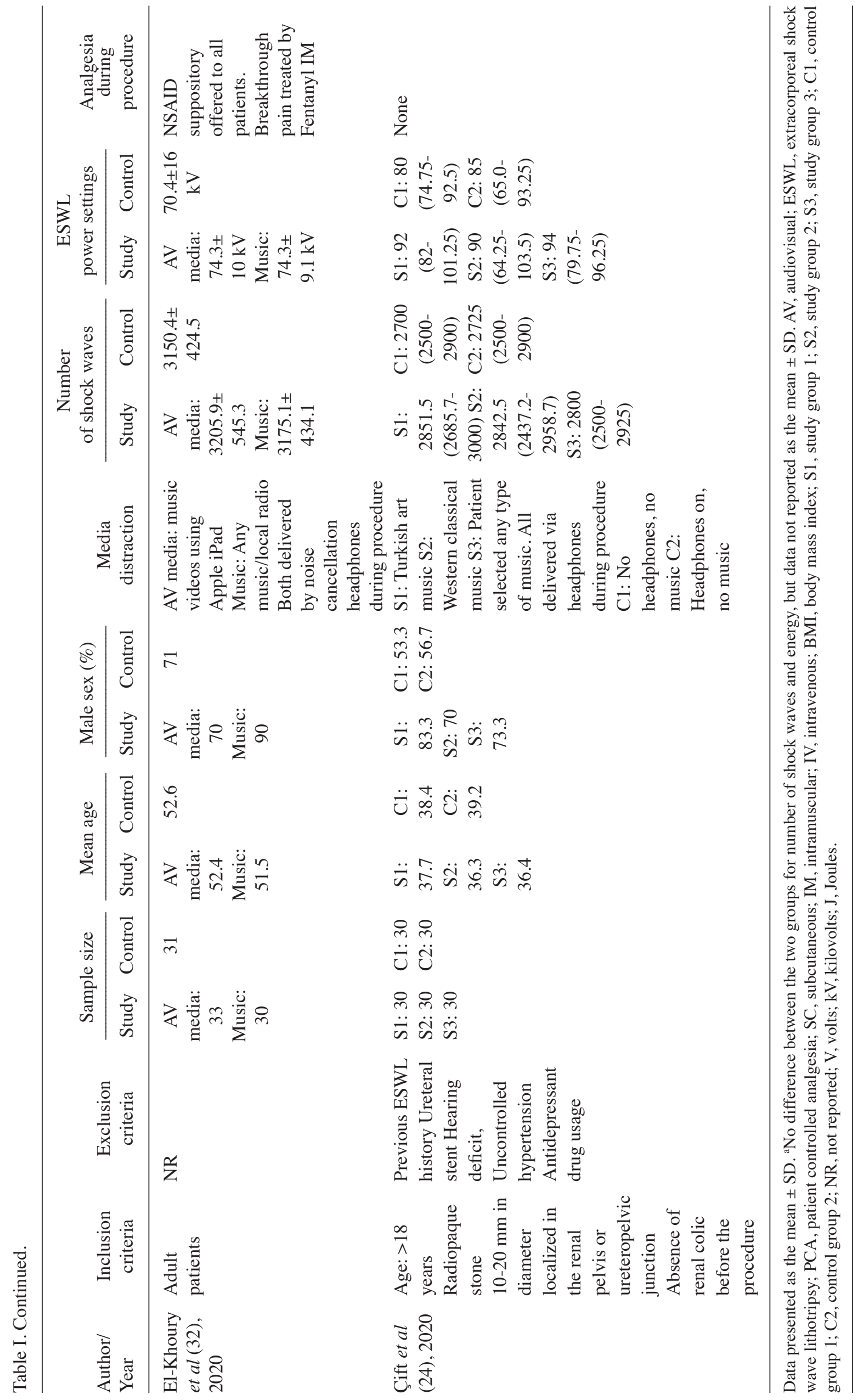




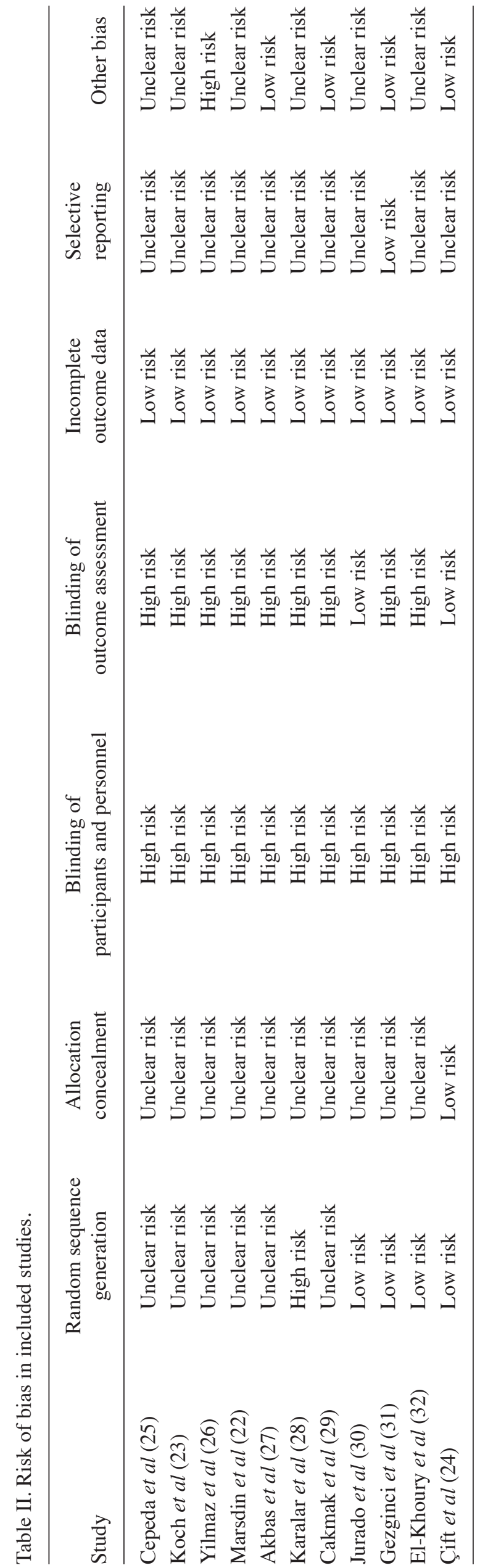

in the two groups using a 10-point VAS scale and reported no statistically significant difference between the two groups.

\section{Discussion}

After a comprehensive review of the literature, the results of the present study indicated that media distraction in the form of AV media or music may lead to a statistically significant reduction of pain and anxiety in patients undergoing ESWL. Data from a limited number of studies also indicate that the use of noise-canceling headphones may lead to a significant reduction in pain and that such an effect may not be seen with regular headphones.

Several non-pharmacological methods of pain management, including massage, relaxation therapy, acupuncture, hypnosis, aromatherapy, music and AV distraction, have been used for a variety of healthcare-procedures worldwide $(33,34)$. Music interventions for pain management can be in the form of music therapy, where a trained professional selects tempo-controlled melodies to have a calming effect, or in the form of random music listening which acts as a distraction tool similar to AV aids $(35,36)$. Owing to its ease of application, a number of studies have assessed the efficacy of such music and/or AV distraction for different medical and surgical procedures. In a recent systematic review and meta-analysis of nine RCTs, Song et al (11) have demonstrated significantly reduced pain and anxiety with the use of music in patients undergoing biopsy. Another RCT has demonstrated that listening to music during labor significantly reduces pain and anxiety of mothers during all stages of labor (37). Similarly, AV aids are effective to reduce pain in patients with sickle cell disease (38), those undergoing colonoscopy (12), as well as those undergoing minor surgeries (39).

The application of media distraction, specifically music, during urological procedures has been evaluated by several trials with conflicting results. Chang et al (40) in a pilot RCT found music to be effective in reducing pain, discomfort, and dissatisfaction in patients undergoing transrectal ultrasound (TRUS)-guided prostate biopsy, however, no such effect was noted in a similar trial by Packiam et al (41). Music has also been used to reduce patient-reported pain and anxiety for other urological procedures, including percutaneous nephrostomy tube placement and urodynamic studies $(42,43)$. As different urological procedures entail different degrees of pain, the current review was focused solely on ESWL to ensure reduced bias owing to non-homogenous procedures. Pain during ESWL can be attributed to several reasons, including trauma caused by shock waves passing through skin and muscles, stretching of the renal capsule where therapy is delivered, or due to an increase in the internal pressure of the kidney (7). Many factors can influence the degree of pain, including the dimensions and position of the urinary stone, frequency of shock waves, power settings, sex and the pain threshold of the patient and use of pre-procedural analgesic (44). As all included studies were RCTs, it is hypothesized that the effect of such baseline variables on outcomes is negated.

The results of the present analysis indicate that media distraction may significantly reduce pain and anxiety scores in patients undergoing ESWL. Similar results were obtained by Kyriakides et al (15) in their meta-analysis of the role 


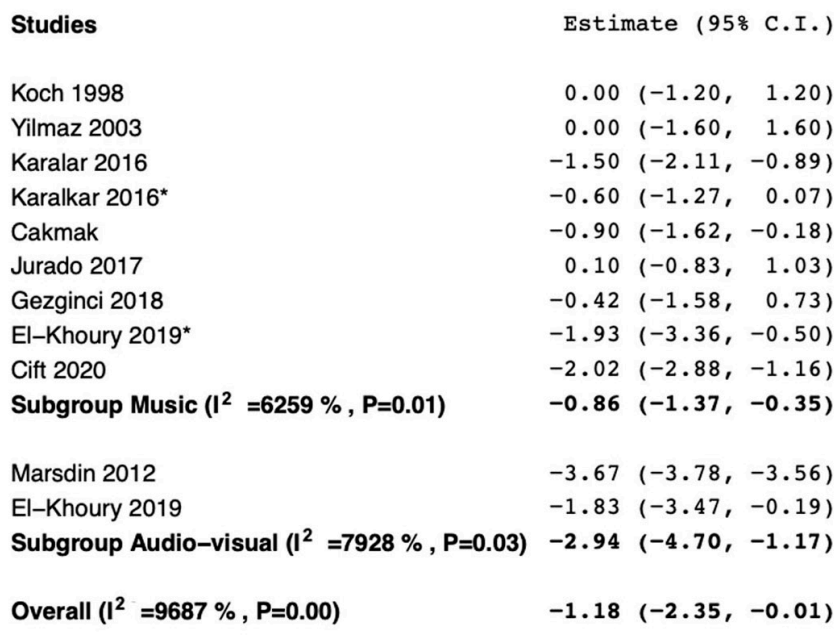

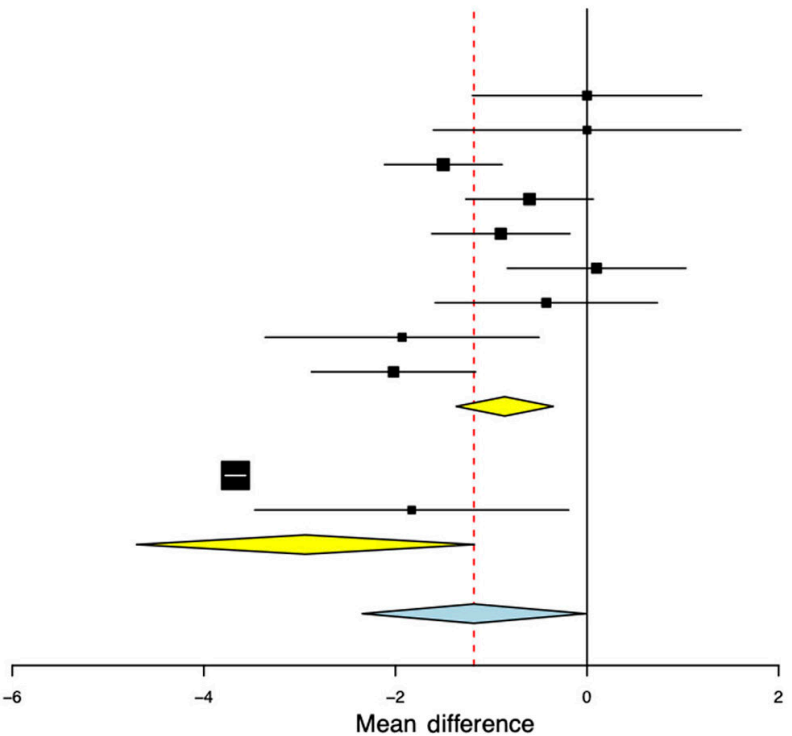

Figure 2. Forest plot of studies comparing media distraction vs. control for the outcome pain with sub-group analysis based on the type of media distraction (audiovisual or music). "Indicates different sub-group from the same study.

\begin{tabular}{lrlr} 
Studies & \multicolumn{2}{l}{ Estimate $(95 \%$ C.I. $)$} \\
Overall & -1.18 & $(-2.35,-0.01)$ \\
& & & \\
- Koch 1998 & -1.30 & $(-2.49,-0.10)$ \\
- Yilmaz 2003 & -1.29 & $(-2.49,-0.08)$ \\
- Marsdin 2012 & -0.92 & $(-1.40$, & $-0.43)$ \\
- Karalar 2016 & -1.14 & $(-2.44$, & $0.15)$ \\
- Karalkar 2016* & -1.24 & $(-2.44$, & $-0.04)$ \\
- Cakmak 2017 & -1.21 & $(-2.44$, & $0.02)$ \\
- Jurado 2017 & -1.31 & $(-2.49$, & $-0.14)$ \\
- Gezginci 2018 & -1.26 & $(-2.47$, & $-0.04)$ \\
- El-Khoury 2019 & -1.12 & $(-2.35$, & $0.11)$ \\
- El-Khoury 2019* & -1.11 & $(-2.35$, & $0.13)$ \\
- Cift 2020 & -1.09 & $(-2.37$, & $0.18)$
\end{tabular}

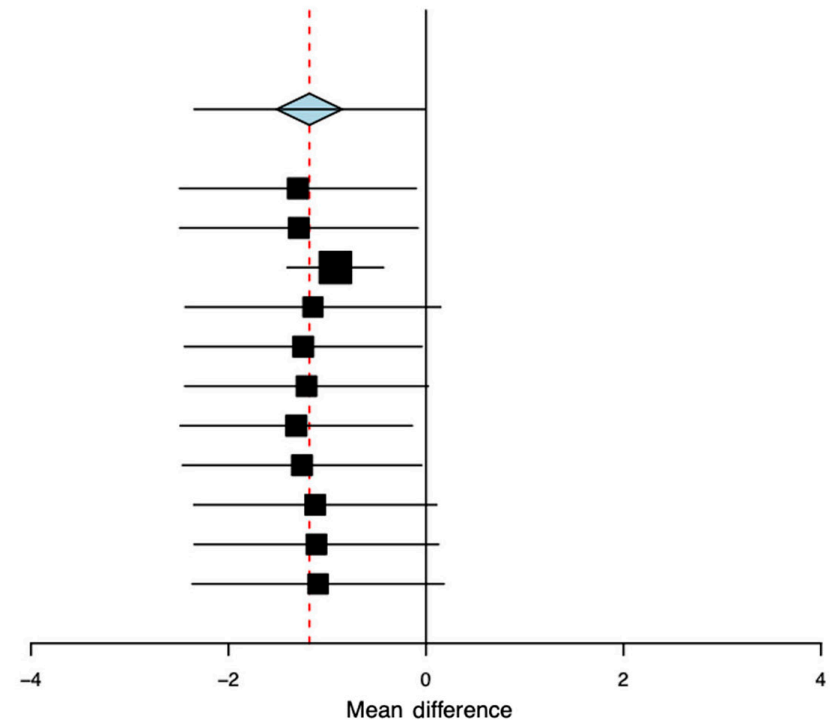

Figure 3. Sensitivity analysis of pain outcome presenting the resultant effect size after exclusion of one study at a time. Black squares in front of each study indicate the effect size and horizontal lines indicate the $95 \%$ confidence intervals, after exclusion of that study from the analysis. "Indicates different sub-group from the same study.

of music in reducing pain and anxiety for any urological procedure. Compared to the present review, they focused only on music and were able to include only six studies for ESWL. The present results also concur with a very recent systematic review of Saraogi et al (45), which indicates that complementary medicine strategies including music, acupressure, acupuncture, transcutaneous electrical nerve stimulation and audiovisual distractions can reduce pain and anxiety in patients undergoing ESWL.

In a sub-group analysis based on the type of media, both $\mathrm{AV}$-media and music were found to significantly reduce pain. However, results should be interpreted with caution, as only two studies were available for the sub-group analysis of AV media. Also, relevant data on anxiety were reported by only five studies. While assessing the results of patient-reported outcomes, it is important to bear in mind the concept of minimal clinically important difference (MCID). The MCID is defined as the smallest change in a measurement that signifies an important improvement in a symptom (46). Data from non-urological studies indicates that the MCID for the VAS scale is 1.4 while for STAI-SA is $10(47,48)$. The pooled MD of VAS scores of all studies in the present analysis was -1.18 (95\% CI: $-2.35,-0.01)$, for studies on music was -0.86 (95\% CI: $-1.37,-0.35$ ) and for STAI-SA scores was -3.91 (95\% CI: -6.44, -1.38). Interpreted in the context of the reported MCID, it can be noted that the effect size of the intervention was small and the upper limits of 95\% CI were close to zero for both pain and anxiety outcomes. This can have important clinical implications as, even though studies suggest a significant difference in pain and anxiety 


\begin{tabular}{|c|c|c|}
\hline Studies & Estimate $(95 \%$ & \& C.I.) \\
\hline Koch 1998 & $0.00(-1.20$, & $1.20)$ \\
\hline Yilmaz 2003 & $0.00(-1.60$ & $1.60)$ \\
\hline Marsdin 2012 & $-3.67(-3.78$ & $-3.56)$ \\
\hline Karalkar $2016^{\star}$ & $-0.60(-1.27$ & 0.07 ) \\
\hline Cakmak 2017 & $-0.90(-1.62$ & $-0.18)$ \\
\hline Gezginci 2018 & $-0.42(-1.58$ & $0.73)$ \\
\hline Cift 2020 & $-2.02(-2.88$ & $-1.16)$ \\
\hline Subgroup No-NCH $\left(I^{2}=9729 \%, P=0.00\right)$ & $-1.13(-2.66$ & $0.40)$ \\
\hline Karalar 2016 & $-1.50(-2.11$ & $-0.89)$ \\
\hline Jurado 2017 & $0.10(-0.83$ & $1.03)$ \\
\hline El-Khoury 2019 & $-1.83(-3.47$ & $-0.19)$ \\
\hline El-Khoury $2019^{\star}$ & $-1.93(-3.36$ & $-0.50)$ \\
\hline Subgroup NCH $\left(1^{2}=6989 \%, P=0.02\right)$ & $-1.20(-2.17$ & $-0.23)$ \\
\hline Overall $\left(I^{2}=9687 \%, P=0.00\right)$ & $-1.18(-2.35$ & $-0.01)$ \\
\hline
\end{tabular}

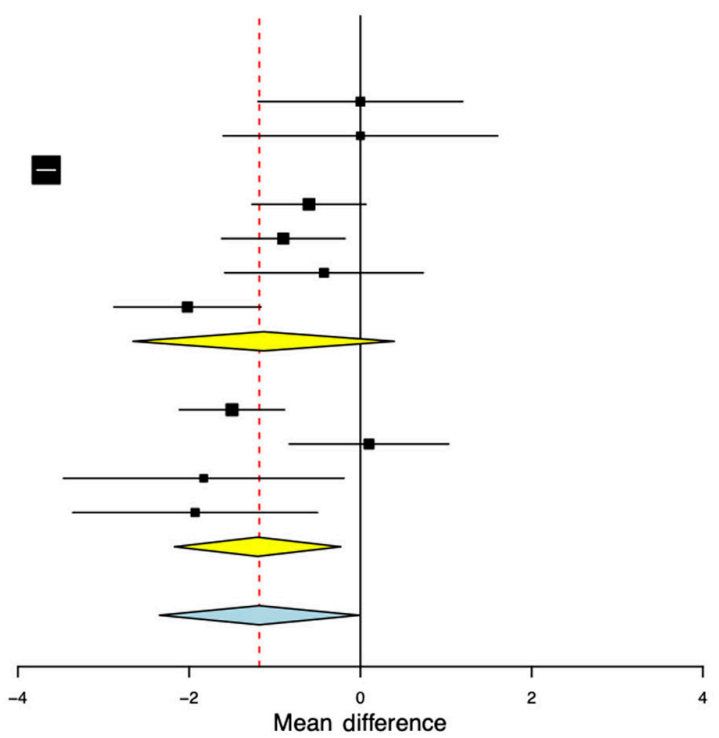

Figure 4. Forest plot of studies comparing media distraction vs. control for the outcome pain with sub-group analysis based on the use of noise-cancelling headphones $(\mathrm{NCH})$. "Indicates different sub-group from the same study.

\begin{tabular}{lrrr} 
Studies & \multicolumn{2}{c}{ Estimate $(95 \%$ C.I.) } \\
Yilmaz 2003 & -0.44 & $(-1.80$, & $0.92)$ \\
Karalar 2016 & -9.60 & $(-13.68$, & $-5.52)$ \\
Karalkar 2016 & -4.20 & $(-9.30$, & $0.90)$ \\
Cakmak 2017 & -3.30 & $(-5.48$, & $-1.12)$ \\
Gezginci 2018 & -4.15 & $(-8.86$, & $0.56)$ \\
Cift 2020 & -3.87 & $(-6.91$, & $-0.83)$ \\
& & & \\
Overall $(\mathbf{1 2}=\mathbf{7 7 7 3} \%, \mathbf{P}<\mathbf{0 . 0 1})$ & $\mathbf{- 3 . 9 1}$ & $(-\mathbf{6 . 4 4}$, & $\mathbf{- 1 . 3 8})$
\end{tabular}

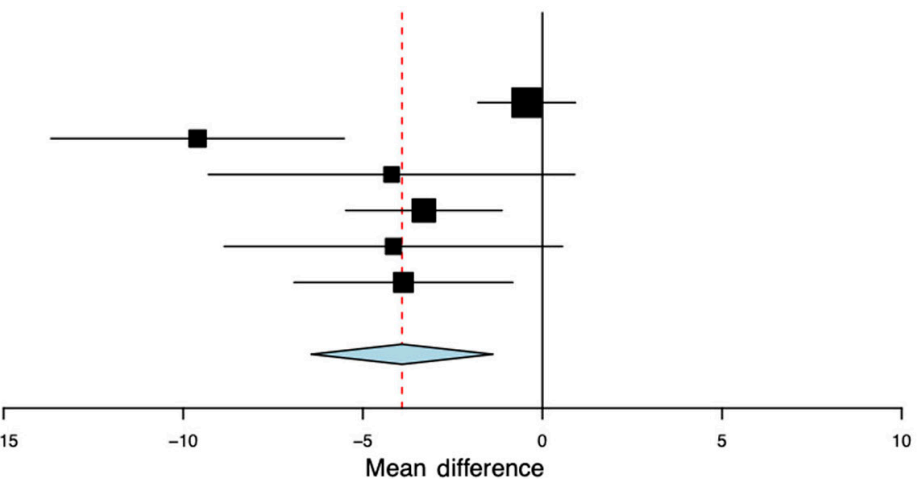

Figure 5. Forest plot of studies comparing media distraction vs. control for the outcome anxiety.

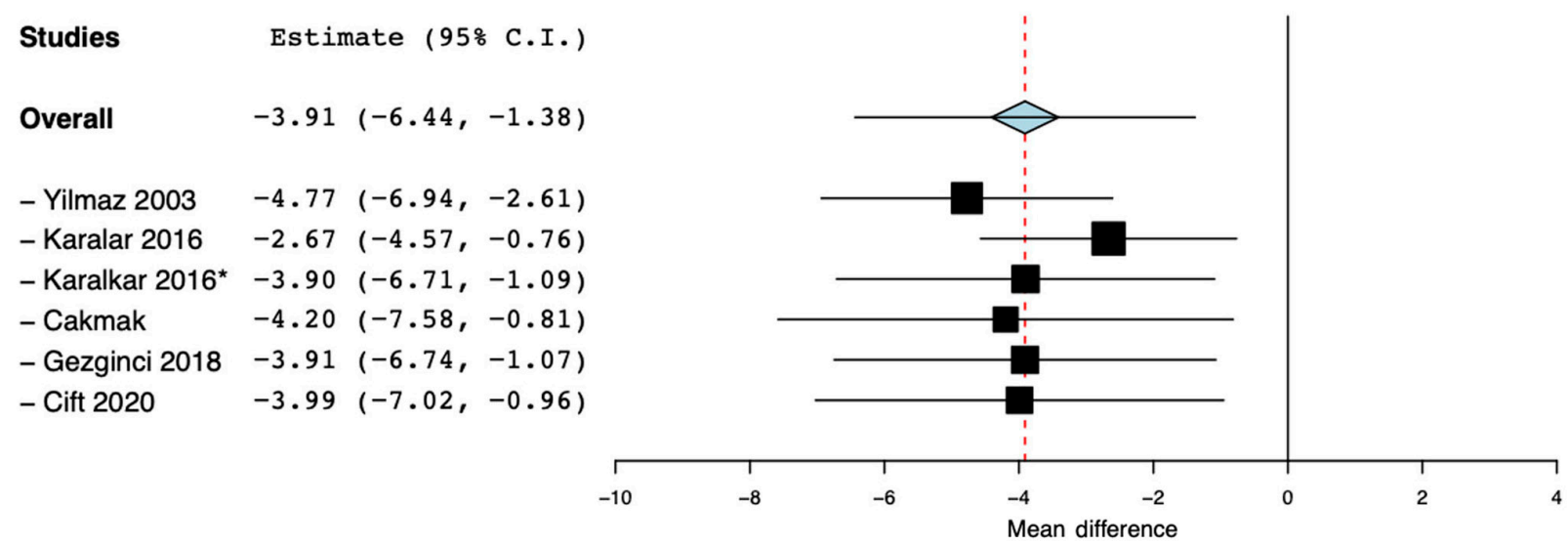

Figure 6. Sensitivity analysis of anxiety outcome presenting the resultant effect size after exclusion of one study at a time."Indicates different sub-group from the same study.

outcomes with media distraction, the resultant effect may not produce a clinically significant improvement in symptoms.

In another sub-group analysis, the present results indicated a significant reduction in pain scores with the use of noise cancellation headphones, but no such effect was noted with regular headphones. Since ESWL is a noisy procedure, the continuous unpleasant sound of shockwaves may affect patient comfort and anxiety (28). Therefore, active noise control employed by such noise-cancellation headphones may lend a more comfortable environment for ESWL thereby influencing 
pain outcomes. However, since only three studies utilized this technology, results should be interpreted with caution.

The limitations of the present review should be mentioned. Firstly, the quality of the included studies in the present review was not high. The absence of adequate methods of randomization and lack of blinding, due to the nature of the intervention, may have influenced outcomes. Secondly, there were methodological variations amongst included studies for the type of music/AV media, choice of music, use of pre-procedural analgesics and patient-controlled analgesia. This may have contributed to the high heterogeneity in the present meta-analyses. Thirdly, data from two studies were not available for the primary outcome and only five studies were pooled for the secondary outcome, which has limited the power of the present analyses. Further, all studies assessing the role of media distraction on anxiety utilized only music as the intervention. The role of AV media on anxiety scores could not be assesses. Fourthly, due to a limited number of studies in the analysis and limitations of the meta-analysis software, publication bias could not be assessed in the present review. Lastly, the results for pain were not stable on sensitivity analysis, as the exclusion of two studies resulted in non-significant results.

The present study has its strengths in being the first meta-analysis focused on the role of media distraction during ESWL. Only RCTs were analyzed to provide level-1 evidence. Sensitivity and subgroup analysis were performed to assess the influence of each study on the effect size and to account for inter-study variations.

To conclude, the present study indicates that media distraction in the form of AV media or music may be beneficial in reducing the pain and anxiety of patients undergoing ESWL. The use of noise-canceling headphones to deliver the intervention may have some benefit over regular headphones. Evidence is, however, weak considering the small effect size, confidence intervals being close to zero and instability of the results on sensitivity analysis. In clinical practice, media distraction may be used during ESWL as a nursing intervention, but a clinically important reduction of pain and anxiety may not be expected.

\section{Acknowledgements}

Not applicable.

\section{Funding}

No funding was received.

\section{Availability of data and materials}

The datasets used and/or analyzed during the current study are available from the corresponding author on reasonable request.

\section{Authors' contributions}

WH conceived and designed the study. KY, LZ and XL collected the data, performed the literature search and were responsible for confirming the authenticity of all the raw data. WH was involved in the writing of the manuscript. All authors have read and approved the final manuscript.

\section{Ethical approval}

Not applicable.

\section{Patient consent for publication}

Not applicable.

\section{Competing interests}

The authors declare that they have no competing interests.

\section{References}

1. Amato M, Lusini ML and Nelli F: Epidemiology of nephrolithiasis today. Urol Int 72 (Suppl 1): S1-S5, 2004.

2. Raheem OA, Khandwala YS, Sur RL, Ghani KR and Denstedt JD: Burden of urolithiasis: Trends in prevalence, treatments, and costs. Eur Urol Focus 3: 18-26, 2017.

3. Kim CH, Shin DS, Kim TB and Jung H: The efficacy of early extracorporeal shockwave lithotripsy for the treatment of ureteral stones. Urol J 16: 331-336, 2019.

4. Gupta NP and Kumar A: Analgesia for pain control during extracorporeal shock wave lithotripsy: Current status. Indian J Urol 24: 155-158, 2008.

5. Chaussy C, Schmiedt E, Jocham D, Brendel W, Forssmann B and Walther V: First clinical experience with extracorporeally induced destruction of kidney stones by shock waves. J Urol 127: 417-420, 1982.

6. Bach C, Zaman F, Kachrilas S, Kumar P, Buchholz N and Masood J: Drugs for pain management in shock wave lithotripsy. Pain Res Treat 2011: 259426, 2011

7. Ozsaker E and Diramali A: The effect of transcutaneous electrical nerve stimulation for pain relief during extracorporeal shock-wave lithotripsy procedure. Pain Manag Nurs 15: 59-68, 2014.

8. Brown MA: The role of nurses in pain and palliative care. J Pain Palliat Care Pharmacother 27: 300-302, 2013.

9. Zins S, Gross C and Hooke MC: Complementary therapies for pain among individuals receiving hemodialysis: A systematic review. Nephrol Nurs J 45: 13-23, 2018.

10. Aydin D, Sahiner NC and Ciftci EK: Non-pharmacological strategies used to reduce procedural pain in infants by nurses at family health centres. J Pak Med Assoc 67: 889-894, 2017.

11. Song M, Li N, Zhang X, Shang Y, Yan L, Chu J, Sun R and Xu Y: Music for reducing the anxiety and pain of patients undergoing a biopsy: A meta-analysis. J Adv Nurs 74: 1016-1029, 2018.

12. Xiaolian J, Xiaolin L and Lan ZH: Effects of visual and audiovisual distraction on pain and anxiety among patients undergoing colonoscopy. Gastroenterol Nurs 38: 55-61, 2015.

13. Drahota A, Galloway E, Stores R, Ward D, Severs M and Dean T: Audiovisual distraction as an adjunct to pain and anxiety relief during minor surgery. Foot (Edinb) 18: 211-219, 2008.

14. Sahiner NC and Bal MD: The effects of three different distraction methods on pain and anxiety in children. J Child Health Care 20: 277-285, 2016.

15. Kyriakides R, Jones P, Geraghty R, Skolarikos A, Liatsikos E, Traxer O, Pietropaolo A and Somani BK: Effect of music on outpatient urological procedures: A systematic review and meta-analysis from the european association of urology section of uro-technology. J Urol 199: 1319-1327, 2018.

16. Goh NM, Drake T, Rai BP and Somani BK: Complementary approaches to decreasing discomfort during shockwave lithotripsy (SWL). Urolithiasis 42: 189-193, 2014.

17. Moher D, Liberati A, Tetzlaff J and Altman DG; PRISMA Group: Preferred reporting items for systematic reviews and meta-analyses: The PRISMA statement. PLoS Med 6: e1000097, 2009.

18. Higgins J, Thomas J, Chandler J, Cumpston M, Li T, Page M and Welch V (eds): Cochrane handbook for systematic reviews of interventions. Version 6. Cochrane, 2019.

19. Elwood LS, Wolitzky-Taylor K and Olatunji BO: Measurement of anxious traits: A contemporary review and synthesis. Anxiety Stress Coping 25: 647-666, 2012.

20. Wallace BC, Schmid CH, Lau J and Trikalinos TA: Meta-analyst: Software for meta-analysis of binary, continuous and diagnostic data. BMC Med Res Methodol 9: 80, 2009. 
21. Wan X, Wang W, Liu J and Tong T: Estimating the sample mean and standard deviation from the sample size, median, range and/or interquartile range. BMC Med Res Methodol 14: 135, 2014.

22. Marsdin E, Noble JG, Reynard JM and Turney BW: Audiovisual distraction reduces pain perception during shockwave lithotripsy. J Endourol 26: 531-534, 2012.

23. Koch ME, Kain ZN, Ayoub C and Rosenbaum SH: The sedative and analgesic sparing effect of music. Anesthesiology 89: 300-306, 1998

24. Çift A and Benlioglu C: Effect of different musical types on patient's relaxation, anxiety and pain perception during shock wave lithotripsy: A randomized controlled study. Urol J 17: 19-23, 2020.

25. Cepeda MS, Diaz JE, Hernandez V, Daza E and Carr DB: Music does not reduce alfentanil requirement during patient-controlled analgesia (PCA) use in extracorporeal shock wave lithotripsy for renal stones. J Pain Symptom Manage 16: 382-387, 1998.

26. Yilmaz E, Ozcan S, Basar M, Basar H, Batislam E and Ferhat M: Music decreases anxiety and provides sedation in extracorporeal shock wave lithotripsy. Urology 61: 282-286, 2003.

27. Akbas A, Gulpinar MT, Sancak EB, Karakan T, Demirbas A Utangac MM, Dede O, Sancaktutar AA, Simsek T, Sahin B and Resorlu B: The effect of music therapy during shockwave lithotripsy on patient relaxation, anxiety, and pain perception. Ren Fail 38: 46-49, 2016

28. Karalar M, Keles I, Doğantekin E, Kahveci OK and Sarici H: Reduced pain and anxiety with music and noise-canceling headphones during shockwave lithotripsy. J Endourol 30: 674-677, 2016.

29. Cakmak O, Cimen S, Tarhan H, Ekin RG, Akarken I, Ulker V, Celik O, Yucel C, Kisa E, Ergani B, et al: Listening to music during shock wave lithotripsy decreases anxiety, pain, and dissatisfaction: A randomized controlled study. Wien Klin Wochenschr 129: 687-691, 2017.

30. Ordaz Jurado DG, Budia Alba A, Bahilo Mateu P, Trassierra Villa M, López-Acón D and Boronat Tormo F: Litotricia por ondas de choque con música: Un tratamiento menos doloroso y más satisfactorio/Shockwave lithotripsy with music: Less painful and more satisfactory treatment. Actas Urol Esp 41: 584-589, 2017.

31. Gezginci E, Iyigun E, Yalcin S, Bedir S and Ozgok IY: Comparison of two different distraction methods affecting the level of pain and anxiety during extracorporeal shock wave lithotripsy: A randomized controlled trial. Pain Manag Nurs 19: 295-302, 2018.

32. El-Khoury HJ, Brookes JDL, Tan B, Shahbaz S and McCahy PJ: Impact of modern media on analgesic requirements during extracorporeal shockwave lithotripsy. ANZ J Surg 90: 53-56, 2020.

33. Smith CA, Levett KM, Collins CT, Armour M, Dahlen HG and Suganuma M: Relaxation techniques for pain management in labour. Cochrane Database Syst Rev 3: CD009514, 2018.

34. Lin YC, Wan L and Jamison RN: Using integrative medicine in pain management: An evaluation of current evidence. Anesth Analg 125: 2081-2093, 2017.

35. Bradt J, Potvin N, Kesslick A, Shim M, Radl D, Schriver E, Gracely EJ and Komarnicky-Kocher LT: The impact of music therapy versus music medicine on psychological outcomes and pain in cancer patients: A mixed methods study. Support Care Cancer 23: 1261-1271, 2015.

36. Gold C, Erkkilä J, Bonde LO, Trondalen G, Maratos A and Crawford MJ: Music therapy or music medicine? Psychother Psychosom 80: 304-305, 2011.
37. Simavli S, Gumus I, Kaygusuz I, Yildirim M, Usluogullari B and Kafali H: Effect of music on labor pain relief, anxiety level and postpartum analgesic requirement: A randomized controlled clinical trial. Gynecol Obstet Invest 78: 244-250, 2014.

38. Ezenwa MO, Yao Y, Engeland CG, Molokie RE, Wang ZJ, Suarez ML and Wilkie DJ: A randomized controlled pilot study feasibility of a tablet-based guided audio-visual relaxation intervention for reducing stress and pain in adults with sickle cell disease. J Adv Nurs 72: 1452-1463, 2016.

39. Hudson BF and Ogden J: Exploring the impact of intraoperative interventions for pain and anxiety management during local anesthetic surgery-a systematic review and meta-analysis. J Perianesth Nurs 31: 118-133, 2016.

40. Chang YH, Oh TH, Lee JW, Park SC, Seo IY, Jeong HJ and Kwon WA: Listening to music during transrectal ultrasound-guided prostate biopsy decreases anxiety, pain and dissatisfaction in patients: A pilot randomized controlled trial. Urol Int 94: 337-341, 2015.

41. Packiam VT, Nottingham CU, Cohen AJ, Eggener SE and Gerber GS: No effect of music on anxiety and pain during transrectal prostate biopsies: A randomized trial. Urology 117: 31-35, 2018.

42. Hamidi $\mathrm{N}$ and Ozturk $\mathrm{E}$ : The effect of listening to music during percutaneous nephrostomy tube placement on pain, anxiety, and success rate of procedure: A randomized prospective study. J Endourol 31: 457-460, 2017.

43. Diri MA, Çetinkaya F and Gül M: The effects of listening to music on anxiety, pain, and satisfaction during urodynamic study: A randomized controlled trial. Urol Int 103: 444-449, 2019.

44. Kumar A, Gupta NP, Hemal AK and Wadhwa P: Comparison of three analgesic regimens for pain control during shockwave lithotripsy using dornier delta compact lithotripter: A randomized clinical trial. J Endourol 21: 578-582, 2007.

45. Saraogi M, Geraghty RM, Hameed B, Rob S, Pietropaolo A, Sarica K, Gozen A, Liatsikos E and Somani BK: Role of complementary medicine (music, acupuncture, acupressure, TENS and audio-visual distraction) in shockwave lithotripsy (SWL): A systematic review from EAU sections of urolithiasis (EULIS) and uro-technology (ESUT). Urology 145: 38-51, 2020.

46. Tashjian RZ, Deloach J, Porucznik CA and Powell AP: Minimal clinically important differences (MCID) and patient acceptable symptomatic state (PASS) for visual analog scales (VAS) measuring pain in patients treated for rotator cuff disease. J Shoulder Elbow Surg 18: 927-932, 2009.

47. Delgado DA, Lambert BS, Boutris N, McCulloch PC, Robbins AB, Moreno MR and Harris JD: Validation of digital visual analog scale pain scoring with a traditional paper-based visual analog scale in adults. J Am Acad Orthop Surg Glob Res Rev 2: e088, 2018.

48. Corsaletti BF, Proença M-DGL, Bisca GKW, Leite JC, Bellinetti LM and Pitta F: Minimal important difference for anxiety and depression surveys after intervention to increase daily physical activity in smokers. Fisioter E Pesqui 21: 359-364, 2014.

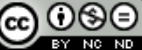

This work is licensed under a Creative Commons Attribution-NonCommercial-NoDerivatives 4.0 International (CC BY-NC-ND 4.0) License. 